\title{
NORMEN HOCHSPRACHLICHEN SPRECHENS ZWISCHEN SCHRIFTSPRACHE UND PLURIZENTRISCHER SPRACHKULTUR
}

\author{
LUDWIG M. EICHINGER
}

\begin{abstract}
Erst seit dem 19. Jahrhundert gewinnt die deutsche Hochsprache in ihrer gesprochenen Form in großen Kreisen der Bevölkerung an Bedeutung. Bis dahin spricht der Großteil der Bevölkerung eine jener regionalen Varietäten des Deutschen, die unter dem Eindruck der Ausbreitung der Hochsprache und von sogenannten Umgangssprachen eine Verschiebung ihrer Funktion mitmachen, als der Hochsprache gegenüberstehender Pol verstanden, so als 'Dialekt' wissenschaftlich beschrieben und ideologisch integriert werden. Spätestens seit der Mitte des 20. Jahrhunderts verändert sich der Sprachgebrauch in eine Richtung, die eine solche dichotomische Einordnung als obsolet erscheinen lässt. In den letzten zwei Jahrzehnten beobachtet man eine beschleunigte weiträumige und tiefgreifende Annäherung an die Standardsprache auch beim Sprechen. Das hat Konsequenzen für die normativen Vorstellungen von solch einer Sprachform, für die das Bild vom plurizentrischen Charakter des Deutschen keine hinreichende Basis mehr abgibt. Eine andere Frage ist, wie sich diese Entwicklungen angemessen modellieren lassen und welche Rolle die Kategorie Regionalität dabei spielt.
\end{abstract}

\section{DIE HISTORISCHE BASIS: SCHRIFTSTANDARD UND REGIONALES SPRECHEN}

\subsection{Drucken und Schreiben ist dezidiert nicht Sprechen}

Regionale Unterschiede spielen im Deutschen eine erhebliche Rolle, sie prägen sowohl den Außeneindruck wie die Wahrnehmung der Sprecher selbst wesentlich stärker als zum Beispiel im Französischen und im Englischen. ${ }^{1}$ Von den größeren europäischen Sprachen mit dem Deutschen vergleichbar ist am ehesten das Italienische, bei dem sich ebenso ein Kompromissstandard entwickelt hat, und wo bis heute die regionalen Unterschiede merklich und bewusst sind. Wie man vom Italienischen sagt, es sei die Sprache der Toskana mit römischer Aussprache, so kann man vom Deutschen mit einer gewissen Berechtigung sagen, es sei eine ostmittelund -oberdeutsche Sprachform im niederdeutschen Mund. Die überregionalen Vereinheitlichungstendenzen seit der frühen Neuzeit - mit

\footnotetext{
${ }^{1}$ Das führt, wie Durrell nachweist, zu unterschiedlichen Konzeptualisierungen von 'Dialekt' und 'Standard'; vgl. Martin Durrell, 'Standardsprache in England und Deutschland', Zeitschiff für Germunistische Linguistik, 27 (1999), S. 285 - 308 (300 - 4).Vgl. auch Martin Durrell, 'Sprachliche Variation als Kommunikationsbarriere', in Deutsch als Fremdsprache. An den Quellen eines Faches. Festschrifl für Gerhard Helbig zum 65. Geburtsiag, hg. v. Heidrun Popp, München 1995, S. 417ff. (S. 422).
} 
den Leistungen Luthers und der Verbreitung der Luther-Bibel als einem markanten Ereignis - laufen auf ein sprachliches System hinaus, das sich als Kompromiss zwischen ostmitteldeutschen und ostoberdeutschen Erscheinungen darstellt. Das heißt konkret historisch: leitende Zentren der Vereinheitlichung waren einerseits die ostmitteldeutsche Kanzleisprache der Wettiner, der sich auch Luther verpflichtet fühlte, und andererseits die Sprache der kaiserlichen Kanzlei, die unter Maximilian I. ihren Sitz in Innsbruck hatte. In den Raum dazwischen fallen auch mit Städten wie Nürnberg oder Augsburg wirtschaftlich mächtige und der humanistischen Öfnung zur Volkssprache zugeneigte Städte, die als wichtige Druckorte starken Einfluss auf den frühen Ausgleich des Deutschen hatten. Der Westen des Sprachgebiets stand längere Zeit diesem sprachlichen Kompromiss etwas ferner. Im norddeutschen Raum befindet sich zu dieser Zeit die mittelniederdeutsche Sprache, die als Sprache der Hanse eine große Zeit gehabt hatte, in einer Phase des Niedergangs, der zentral bedingt war durch die Verschiebung in der Bedeutung der Wirtschaftsräume im Gefolge der Eroberungen und Entdeckungen in der zweiten Hälfte des 15. Jahrhunderts und der sich durch die Reformation und die Verbreitung der (hochdeutschen) Lutherbibel noch beschleunigte.

\subsection{Wie kann man schreiben, wie man spricht, wenn man spricht, wie man schreibt?}

Sowohl in dem Raum, der den sprachlichen Kompromiss des Frühneuhochdeutschen trug, wie in dem vom Niederdeutschen geprägten Areal führt die Ausbildung der hochdeutschen Ausgleichssprache zu einer Trennung zwischen der alltäglich gesprochenen und der neuen Schriftsprache. Dabei geht es ganz zu Beginn sogar eher noch um eine Vereinheitlichung des Druckens; schon eine weiter verbreitete Übung des Schreibens in der neuen Sprachform stellt erst eine nächste Entwicklungsstufe dar. Und erst ganz allmählich bildet sich eine weiter greifende gesprochene Form des Deutschen heraus, die sich an der neuen Schriftsprache orientiert. Es ist das aber in Anbetracht des geringen Prozentsatzes an schreib- und lesefähigen Leuten über längere Zeit die Sprache einer kleinen gebildeten Schicht, die allerdings allmählich in den Städten mehr und mehr an Größe zunahm. Daneben existierten vor allem auf dem Lande die traditionellen Dialekte weiter, wobei die systematische Differenz zwischen der neuen Allgemeinsprache und den ostmitteldeutschen Dialekten zweifellos geringer war als im gesamten oberdeutschen Raum und gar im Raum des Niederdeutschen, das mit seinen küstenkontinentalgermanischen Eigenheiten in der Findung der Ausgleichssprache kaum eine Rolle spielte. In den nächsten Jahrhunderten (dem 17. und dem 18.) kommt dazu, dass politisch-religiöse Differenzen zu einer Fossilisierung bestimmter Eigenheiten führen: Der katholische Süden wird seine eigene Schreibtradition erst im Verlaufe des 18. Jahrhunderts 
aufgeben. ${ }^{2}$ Gleichzeitig konzentriert sich das kulturelle Schwergewicht in dieser Zeit im Raum des Ostmitteldeutschen - Leipzig wird die kulturell bestimmende Stadt. Das führt letztlich zur Durchsetzung jener Norm, die sich in der Interaktion der gebildeten Schichten herausgebildet hat, wobei historisch der Raum des östlichen Mitteldeutschen eine zentrale Rolle spielte. Im gesamten Norden des deutschen Sprachgebiets führte die grundsätzliche Differenz zum Niederdeutschen dazu, dass sich das Hochdeutsche auch als öffentliche Sprechsprache weitaus früher durchsetzte. So ist es nicht überraschend, dass meinungsführende Schreiber (und Sprecher) aus dem Norden und aus dem Süden, oder eigentlich dem Nichtnorden, zu diesen Fragen ihre eigene Ansicht haben. Die folgende Äußerung von Karl Marx zu diesem Thema reflektiert eher die südliche Sicht, der die Lautiermethode des Schreibenlernens ebenso suspekt sein muss wie die dieser Schreibung zugehörige buchstabengerechte Aussprache: 'Schreibe, wie du sprichst, und sprich, wie du schreibst, lehren uns schon die Elementarlehrer.' ${ }^{3}$

Auf jeden Fall setzt sich so das Hochdeutsche bis zum 19. Jahrhundert als geschriebene und in gewissem Ausmaß auch als gesprochene Form durch. Die unterschiedliche Einbettung des Hochdeutschen führt dazu, dass sich im Verlaufe des 19. und 20. Jahrhunderts über den deutschen Sprachraum verteilt zwar gemeinsame Trends ausmachen lassen, aber auch Unterschiede, die bis heute den Sprachgebrauch prägen. Zum einen wird der Gebrauch des Dialekts mehr und mehr zu einem ländlichen Merkmal, generell ist die Beherrschung standardsprachlicher Formen zudem abhängig vom Bildungsgrad. Wegen der unterschiedlichen sprachlichen Voraussetzungen und wegen differierender historischer Entwicklungen bis ins zwanzigste Jahrhundert hinein ist die soziale Einschätzung von Standardund Dialektgebrauch im deutschen Sprachgebiet unterschiedlich verteilt. Generell gilt aber, dass sich mit der zunehmenden räumlichen Mobilität umgangssprachliche Zwischenformen herausbilden, deren wichtiger Status lange Zeit durch die Fixierung der Sprachwissenschaft auf die Endpunkte 'Dialekt' auf der einen und 'Hochsprache' auf der anderen Seite verdeckt geblieben ist, ${ }^{4}$ obwohl diese Dichotomie spätestens seit dem Wachstum der Städte im 19. Jahrhundert eine Konstruktion ist, die nur vom Kampf des Bürgertums um eine Alleinstellungsmerkmal her verständlich ist. So gibt es unterhalb des geschriebenen Standards und einer sich relativ strikt daran

\footnotetext{
${ }^{2}$ Genaueres zu diesen Grenzziehungen bei Martin Durrell, 'Die "Mainlinie" als sprachliche Grenze', in Dialehtgeographie und Dialektologie. Günter Bellmann zum 60. Geburtstag von seinen Schülern und. Freunden, hg. v. Wolfgang Putschke, Werner Veith und Peter Wiesinger, Marburg 1989 (S. 89); vgl. Ludwig M. Eichinger, 'Sprache und Sprachgebrauch im Süden Deutschlands', in Regionale Standards, Sprachvariationen in den deutschsprachigen Ländern, hg. v. Elisabeth Knipf-Komlósi und Nina Berend, Budapest / Pécs 2001, S. $61-94$ (S. 66ff.)

${ }^{3}$ Karl Marx, 'Debatten über Pressfreiheit und Publikation der Landständischen Verhandlungen. Von einem Rheinländer', Rheinische Zeitung, $\mathrm{Nr} 139$ vom 19. Mai 1842, S. $67-77$ (S. 75), <http://www.mlwerke.de/me/me01/me01_066.htm>.

${ }^{4}$ Vgl. Martin Durrell, 'Zum Problem des sprachlichen Kontinuums im Deutschen', Zeitschrift für Germanistische Linguistik, 26 (1998), S. 17 - 30 (S. 20).
} 
orientierenden Mündlichkeit eine Reihe von sprachlichen Realisierungen, die man zumeist als das Kontinuum der Umgangssprache bezeichnet hat und die jetzt als Substandardvarianten erstens den Sprachgebrauch wesentlich mehr prägen als die traditionellen Dialekte, und zweitens auch jetzt erst das systematische Interesse der Linguistik finden.

\section{DLALEKTE UND DIE NEUBEWERTUNG TRADITIONELLER UNTERSCHIEDE}

Aufgrund der geschilderten Verhältnisse ist es nicht überraschend, dass die Dialekte als solche eigentlich erst so recht wahrgenommen werden, wenn sie wirklich einem stark standardsprachlich orientierten Gesamtsystem gegenüberstehen, und das geschieht im Verlaufe des 19 . Jahrhunderts. ${ }^{5}$ So finden sich auf der Ebene literarischer Texte - abgesehen von in dieser Hinsicht 'realistischen' Vorläufern wie Grimmelshausens Der abentheuerliche Simplicissimus Teutsch (wo übrigens der Teufel Niederdeutsch spricht) ${ }^{6}-$ im 19. Jahrhundert die gleichzeitigen sprachlichen Verhältnisse erstmals auch einigermaßen äquivalent widergespiegelt. Das gilt natürlich immer mit der Einschränkung, dass die literarische Gestaltung gesprochener Sprache nie echt gesprochene Sprache darstellt. ${ }^{7}$ Die Verschiedenheit im Gebrauch regionaler Sprachformen in den literarischen Texten spiegelt zweifellos Unterschiede in der gesellschaftlichen und wirtschaftlichen Entwicklung im Norden und Süden Deutschlands wider - wobei mir aus biographischen Gründen erlaubt sei, als Beispiel für den Süden im wesentlichen Bayern heranzuziehen.

Den Konzepten eines bürgerlichen Mittelstands und der sich allmählich entwickelnden städtischen Welt steht als kultureller Pol das ländliche Leben mit seinen einfachen alltäglichen Verrichtungen gegenüber: Die in diesen Verhältnissen auf dem Lande lebenden Menschen hatten weitaus weniger Gelegenheit und aus praktischen Gründen auch weniger Neigung, über die Anhäufung von Bildungskapital diese sprachliche Welt zu verlassen. ${ }^{8}$

\footnotetext{
${ }^{5}$ Eine wichtige Entwicklung in dieser Hinsicht dokumentiert Stephan Elspaß, Sprachgeschichte von unten. Untersuchungen zum geschriebenen Deutsch im 19. Jahrhundert, Tübingen 2005.

${ }^{6}$ Vgl. Eichinger; op. cit.

${ }^{7}$ Klassisches Beispiel dafür Gerhart Hauptmanns 'Weber', wo zudem in der Abstufung der gewăhlten Sprachformen die soziale Kategorisierung der Figuren durch Dialekt oder an die Hochsprache angenäherte Varianten entspricht. Für die ganz anders gelagerten oberdeutschen Verhältnisse vgl, Ludwig Thomas Tragödie 'Magdalena', wo die Personen, unabhängig von der sozialen Einbettung, ihren bairischen Dialekt sprechen. Standardsprachlichkeit wird eher als ein Zeichen sozialer Inkompetenz angesehen, typisch 'preußische' Standardsprachlichkeit zudem als Merkmal norddeutscher Überheblichkeit. Zum Niederschlag dieser Verhältnisse in der heutigen Vorurteilslandschaft des Deutschen vgl. z.B. Ulrich Ammon, Die deutsche Sprache in Deutschland, Österreich und der Schweiz. Das Problem der nationalen Varietäten, Berlin / New York 1995 (S. 508).

${ }^{8} \mathrm{~S}$. die entsprechenden Ausführungen in Hans Ulrich Wehler, Deutsche Gesellschaftsgeschichte. 1815 1845/49, München 1989 (S. 162ff., 490ff., 642ff.), Hans Ulrich Wehler, Deutsche Gesellschaftsgeschichte. $1849-1914$, München 1995 (S. 831ff.).
} 
Zudem wurde diese Welt, je länger je mehr, auch als Korrelat naturverbundenen und natürlichen Lebens angesehen und - nicht zuletzt auch in einer allmählich aufkommenden entsprechenden Literatur, die zum Teil auch dialektale Form annimmt, entsprechend künstlerisch gestaltet. Aus verschiedenen Gründen kam dieser Prozess im Süden Deutschlands, ja im südlichen deutschen Sprachraum insgesamt stärker zum Tragen als in der Mitte und im Norden. Im Norden, um damit zu beginnen, war das Niederdeutsche durch den Wechsel in das 'fremde' Hochdeutsch viel weiter zurückgedrängt als im Süden, wo zwischen den Dialekten und der Hochsprache ein verwandtschaftliches Kontinuum besteht. ${ }^{9}$ Zum zweiten bot im Süden der selbständigere Status der Bauern (im Vergleich z.B. zu den ostelbischen Verhältnissen) und auch die Entdeckung vor allem der Alpen- und Voralpenlandschaft durch den frühen Tourismus, wenn man das schon so nennen will, eine günstigere Projektionsfläche. So entwickelte sich im bairischen Süden ein ganz deutliches ländliches Selbstbewusstsein, das dem städtischen Selbstbewusstsein durchaus die Waage halten konnte. ${ }^{10}$ Das insbesondere auch deswegen, weil der Süden - wie Ostelbien - viel länger agrarisch strukturiert bleibt und so die Verstädterungsprozesse, die ab 1870 in Mitte und Westen Deutschlands dramatisch einsetzen, nur in gedämpfter Form mitmachte. So bilden sich in den großen süddeutschen Städten - und dafür mag zum Beispiel München stehen - eigene stadtdialektale Formen heraus, ${ }^{11}$ die imagemäßig durchaus mit standardsprachlichen Ansprüchen konkurrieren können. ${ }^{12}$ Wie die Ansprüche solch einer Sprachform mit den Erwartungen norddeutscher Standardsprachlichkeit konfligieren, zeigt sich in schön ironisch gebrochener Weise in Thomas Manns Buddenbrooks, wenn von den enttäuschten Erfahrungen von Toni Buddenbrook in der Ehe mit dem Münchner Permaneder berichtet wird, genauer, von den Kommunikationsschwierigkeiten mit ihrer dortigen Köchin:

Und wenn ich 'Frikadellen' sage, so begreift sie es nicht, denn es heißt hier 'Pflanzerln'; und wenn sie 'Karfiol' sagt, so findet sich wohl nicht so leicht ein Christenmensch, der darauf verfällt, daß sie Blumenkohl meint; und wenn ich sage 'Bratkartoffeln', so schreit sie so lange 'Wahs!', bis ich 'Geröhste Kartoffeln' sage. ${ }^{13}$

\footnotetext{
${ }^{9}$ Vgl. dazu die Darstellung in den entsprechenden Artikeln in Varietäten des Deutschen. Regional- und. Umgangssprachen (= Jahrbuch 1996 des Instituts für Deutsche Sprache), hg. v. Gerhard Stickel, Berlin / New York 1997.

${ }^{10} \mathrm{Es}$ ist klar, dass hier die Entwicklungen in der städtischen Arbeiterschaft, die durchaus auch regionale Sprachmerkmale entwickelt, bei Seite gelassen werden.

${ }^{11}$ Vgl. die kurze zusammenfassende Beschreibung in Bernhard Stör, 'Sprachregion München. Beschreibung eines Projekts zur Stadtsprachenforschung', in Dialektgenerationen, Dialehtfunktionen, Sprachwandel, hg. v. Thomas Stehl, Tübingen 1999; daneben Kurt Rein, Sprachregion München. Ergänzungsband zum Sprachatlas von Oberbayern, Heidelberg 2005.

${ }^{12}$ Auf die Bedeutung solcher positiver und negativer Prestigeannahmen weist auch Durrell, 'Standardsprache in England und Deutschland', S. 303.

${ }^{13}$ Hier - typischerweise - zitiert nach Paul Kretschmer, Wortgeographie der hochdeutschen Umgangssprache, Göttingen 1918, S. 1.
} 
Diese stärker dialektgeneigte Entwicklung hat nicht zuletzt damit zu tun, dass das gesamte Sprachverhalten Süddeutschlands durch die politisch kleindeutsche Lösung, die zur Gründung des deutschen Reiches unter Ausschluss von Österreich führte, ein Sprachverhalten, das in Bayern so ähnlich wie in Österreich war, in einen wesentlich nördlicheren Rahmen geriet, wodurch es seither wesentlich 'auffälliger' erscheint. ${ }^{14}$ Die hier geschilderten Verhältnisse gelten aber für Bayern nun über weite Phasen des 19. Jahrhunderts und weit in das 20. Jahrhundert hinein, wenn natürlich aus verschiedenen Gründen (Schule, Zeitungen usw.) im Lauf der Zeit in allen Schichten die Vertrautheit mit der Hochsprache anstieg. So kommt es denn letztlich, dass bis hin fast zum Zweiten Weltkrieg für weite Schichten der bayrischen Bevölkerung der Dialekt die mit Heimatgefühlen verbundene Sprache des Alltags war. Und typisch ist es somit, dass das, was wir jetzt als alten Dialekt betrachten, häufig die Konkreta einer Art von ländlichem Alltag betrifft, der mit der großen Modernisierungswende ab Mitte der 1950er Jahre endgültig ihr Ende gefunden hat. ${ }^{15}$

\section{JAHRZEHNTE DES WANDELS}

\subsection{Modernisierung}

Und so ist es denn weniger, oder allenfalls indirekt, eine Folge von Sprachenpolitik und Sprachsteuerung, von der die Verwendung des Dialekts in seiner traditionellen Form bedroht worden ist: vielmehr ist es die allgemeine Veränderung der Lebensumstände, die den traditionellen Dialektgebrauch in den letzten Jahrzehnten mehr und mehr als marginal erscheinen lässt und hin zu neuen Formen regionalbezogenen Sprechens führt. Veränderung der Umstände, das heißt aber eigentlich, dass sich eine repräsentative Anzahl von Sprechern entschieden hat, ihre sprachliche Identität in anderen als den traditionellen Dialektformen zu suchen. ${ }^{16}$

Zur Umwertung der, modern gesprochen, Dialekt-Hochdeutsch-Diglossie mit großlandschaftlichen Oralisierungsnormen kam es erst ca. 200 Jahre nach

\footnotetext{
${ }^{14}$ Wo hier die Grenzen verlaufen und wie sie bedingt sind, wird in Martin Durrell, 'Die "Mainlinie" als sprachliche Grenze', ausgeführt.

${ }^{15}$ Vgl. dazu z.B. Ludwig M. Eichinger, 'Fachliches Sprechen und Umgangssprache. Am Beispiel der Terminologie der Landarbeit', in Vom Sturz der Diphthonge, hg. v. Albrecht Greule, Franz Xaver Scheurer und Ludwig Zehetner, Tübingen 2000, S. 187 - 95.

${ }^{16}$ Die kultursoziologischen Hintergründe dieser Entwicklung im süddeutschen Kontext werden in Ludwig M. Eichinger, 'Neue Unübersichtlichkeit. Probleme der Beschreibung des gegenwärtigen Dialektgebrauchs', in Probleme der oberdeutschen Dialektologie und Namenkunde, hg. v. Peter Wiesinger; Werner Bauer und Peter Ernst, Wien 1999, S. 40-61, exemplifiziert; grundsätzlicher und an einem im Blickwinkel dieses Beitrags eher untypischen Raum s. Ludwig M. Eichinger, 'Maritime Kultur im südlichen Ostseeraum', in Maritime Kultur und regionale Identitaten - Der südliche Ostseeraum, hg. von Ludwig M. Eichinger und Friedhelm Debus unter Mitarbeit von Albrecht Plewnia, Stuttgart 2006, S. $7-30$.
} 
ihrer Entstehung, als um 1930 neue überlandschaftliche Oralisierungsnormen massenmedial verbreitet wurden und damit überhaupt zum ersten Mal kommunikativ verfügbar wurden. ${ }^{17}$

Nun ist es ja zweifellos nicht sehr überraschend, wenn die Tatbestände, die unmittelbar mit landwirtschaftlicher und handwerklicher Praxis zu tun hatten, die nunmehr seit Jahrzehnten keine Rolle mehr spielt, auch sprachlich nicht mehr beherrscht oder mit umgangssprachlichem Vokabular beschrieben werden. Und man sollte sich auch nicht wundern, dass es allmählich zu einem Wandel des Sprachgebrauchs führt, wenn die Standardsprache nicht mehr eigentlich eine Fremdsprache darstellt, sondern eine sprachliche Alternative, die von Schulunterricht bis Medienkonsum eine vielfältige Präsenz zeigt. ${ }^{18}$ Dabei ist vielleicht noch besonders hervorzuheben, dass mit der stilistischen Lockerung des Medienangebots im Rahmen des Entstehens von Privatsendern und mit der Regionalisierung der Radiosender nicht mehr nur eine standardsprachliche Form mittlerer Offizialität präsentiert wird, sondern dass verschiedene umgangssprachlich getönte, aber nicht sehr engräumig regionsspezifische Varietäten wahrgenommen werden. Das lockert die engeren regionalen Sprachbindungen, gesteigert noch dadurch, wenn ein gewisses Ausmaß an beruflicher Mobilität dazukommt. Alle diese Faktoren haben im Verlauf der letzten drei Jahirzehnte dramatisch an Bedeutung gewonnen, so dass sich auch das kommunikative Profil der meisten Menschen merklich verändert hat. Die Standardsprache kommt näher, der regionale Raum, in dem man sich bewegt, wird größer, man lernt andere sprechsprachliche Optionen kennen: Das führt ohne Zweifel zu großräumigeren Ausgleichsprozessen, in denen kleinräumige Differenzen verschwinden, und bei denen sich auch die Vorstellungen vom Dialekt und seinen Benutzern in vereinfachender Weise verändert haben.

Auch das schlägt sich aber natürlich im Süden und im Norden in leicht unterschiedlicher Weise nieder; die neuen Substandards mit regionaler Charakteristik und dem Anspruch großräumiger Verständlichkeit lassen sich für den ehemals niederdeutschen Norden sowie auch für große Teile (eines lange schon städtisch geprägten) Mitteldeutschlands als vergleichsweise geringfügige Abweichungen von der 'Hochsprache' ansehen. So wird z.B. in einer neueren Handbuchdarstellung das (heutige) Berlinische (BerlinischBrandenburgische) als 'eine Umgangssprache auf standardnahem Niveau' im Wesentlichen durch 10 [!] phonische Merkmale beschrieben, von denen vier zudem ausschließlich an frequente Wörter gebunden seien:

${ }^{17}$ Jürgen Erich Schmidt, 'Die deutsche Standardsprache: Eine Varietät-drei Oralisierungsnormen', in Standardvariation - Wie viel Variation vertrïgt die deutsche Sprache?, hg. v. Ludwig M.Eichinger und Werner Kallmeyer, Berlin / New York 2005, S. 278 - 305 (S. 285).

${ }^{18}$ Die weitreichenden Konsequenzen dieser Veränderungen werden auch bei Martin Durrell, 'Language, Nation and Identity in the German-speaking Countries', in Standard, Variation und Sprachwandel in germanischen Sprachen, hg. v. Christian Fandrych und Reinier Salverda, Tübingen 2007, S. 37 - 57 (S. 51 - 2) konstatiert. 
$k$ statt $c h$ in $i k$, bisken; $t$ statt $s$ in det, wat; $u$ statt au in $u f$, druf; $i$ statt $e i$ in rin (herein). Mittelhochdeutsch ei und ou > lang $e$ und lang o: keen (kein), boom (Baum); Rundung von $i$ zu ü in büme (Birne), fiusch (Fisch); $p$ für $p f$ in kop (Kopf), apel (Apfel) [...] schlieBlich $j$ für $g$ im Anlaut und Inlaut. ${ }^{19}$

Dazu kämen in der Lexik die 'sprichwörtlich flotten, oft kurzlebigen Neologismen'. Man mag durchaus zu einem anderen Schluss kommen, was die objektive Standardnähe der damit beschriebenen Form angeht, allerdings ist unverkennbar, dass damit eine Form beschrieben wird, die weniger einer traditionellen - und gar ländlichen - Region zugerechnet wird, vielmehr Prestige aus der Vertretung in einer großstädtischen Sprechweise zieht. Im Hinblick auf wirklich moderne Sprechhaltungen kann diese Form allerdings kaum mehr als standardsprachlich gelten.

Auch eine weiträumigere nord- und mitteldeutsche Substandardvariante lasse sich durch entsprechend wenige, und kaum spezifisch Raum bildende - und auch kaum Verständnis hemmende - Merkmale beschreiben.

Aussprache $e$ für langes $\ddot{a}$ in Medchen, Hefen (Häfen) etc.; Aussprache von $e i$, $a u, e u$ jeweils mit längerem Ton auf dem ersten Vokal; kurzer Vokal in Rad, Bad, Glas; ch statt $g$ im freien und gedeckten Auslaut: mach (mag), fliecht (fliegt) etc.; $f$ statt $p f$ im Anlaut: fosten (Pfosten), fanne (Pfanne) etc.; zudem Tendenz zu dingk statt Ding etc. und zum Konsonantenschwund im Auslaut: sin (sind), do (doch), ma (mal). ${ }^{20}$

Wie auch immer das im einzelnen sein mag, es gibt offenbar in Nord- und im nördlichen Mittteldeutschland - aufgrund einer nur ganz allgemeinen Fernwirkung der (v. a. niederdeutschen) Dialekte und der gemeinsamen sprechsprachlichen Verarbeitung einer schriftsprachlichen Vorgabe offenbar einen relativ begrenzten Satz von variierenden Merkmalen, denen man, wenn man will, ggf. noch stilistisch gemeinte regionale Highlights aufsetzen kann, z.B. getrennte Aussprache von st in Hamburg und Umgebung, insgesamt noch 'norddeutscher' z.B. starke Vokalisierung des postvokalischen r: faht (Fahrt), hüasch (Hirsch), deutliche $t$-Epenthese bei ebent (eben). Auffälligere Merkmale, mit denen sicher auch eine weit gefasste Übergangszone des Standards verlassen wäre, wären allerdings schon die Diphthongierung halbhoher Langvokale: bouden (Boden), meiter (Meter) ggf. gekoppelt mit Lenisierung der inlautenden Tenues: meider (Meter), auch $s p$ getrennt gesprochen und Monophthong: speel (Spiel).

Im Einzelnen ungeklärt ist, an welcher Stelle - bei welcher Kombination von Merkmalen - ein nächster (nicht mehr standardnaher) Substandard

\footnotetext{
${ }^{19}$ Werner Besch, 'Territoriale Differenzierung', in Kleine Enzyklopädie Deutsche Sprache, hg. v. Wolfgang Fleischer, Gerhard Helbig und Gotthard Lerchner, Frankfurt a.M. 2001, S. $383-423$ (S. 420-1).

${ }^{20}$ Besch, op. cit., S. 421.
} 
abzugrenzen ist. ${ }^{21}$ Die jeweilige Entscheidung für den Ort dieser Grenze ist ganz offenbar davon abhängig, wie viele Merkmale von medialer Mündlichkeit als strukturelle Merkmale akzeptiert werden. Daneben gibt es offenbar aufbauend auf den modernen Sprechererfahrungen eine Annäherung an einen normativen Konsens über die Salienz regionaler Merkmale, die zu Makrosynchronisierungen ${ }^{22}$ führt, auf deren Basis Schriftsprachnähe der Artikulation bzw. die dazu erlernten Parallelisierungserfahrungen von orthographischer und orthoepischer Regel eine zentrale Rolle spielen.

Es ist sicherlich nicht zufällig, dass sich die Beschreibungen im Wesentlichen auf Lautliches - mit Ausblicken auf das Lexikon - beziehen. ${ }^{23}$ Dabei hat sich nicht zuletzt mit dem Erscheinen des 'Variantenwörterbuchs', ${ }^{24}$ das die Frage der Standardwertigkeit mit der der nationalen Varietäten verbindet, die Basis der einschlägigen Diskussion deutlich verbreitert. Es ist nach Durchsicht dieser Zusammenstellung viel weniger klar, was hier jeweils als Standard gilt, und viel klarer, dass plurizentrische Organisation und nationale Varietäten nicht dasselbe sind. ${ }^{25}$

Im Bereich der eigentlichen Grammatik sind wir offenbar weniger kompromissbereit, was unsere Standardansprüche angeht (im Folgenden ein Teil der Beispiele nach Besch 2001). Das betrifft für norddeutsche Substandards die Folgen des Kasuszusammenfalls im Akkusativ (Akkudativ). Spätfolge davon ist zum Beispiel der viel bespöttelte \$atz einer bundesdeutschen Fernsehberühmtheit: Da werden Sie geholfen (Da hilft man Ihnen, ggf.: Da bekommen Sie geholfen).

Relativ starken Regionalitätscharakter haben auch die sehr norddeutschen (küstenwestgermanisch geprägten) Möglichkeiten des 'preposition stranding':

da halte ich nichts von (davon halte ich nichts); da kann ich nichts an mit fangen (damit kann ich nichts anfangen) oder auch bestimmter Verwendungen der tun-Periphrase:

Ich find das ja nu irgendwie taktlos, dass man den mitten (mit einem) Ungeheuer vergleichen tut.

${ }^{21}$ Ohne dass damit der Eindruck erweckt werden sollte, es handle sich hier um strikt distinkte Varianten.

${ }^{22}$ Terminus nach Jürgen Erich Schmidt, op. cil, S. 278 - 305, wo auch die empirische Stützung dieser Tendenz zumindest für Teile des mittel- und niederdeutschen Raums geleistet wird.

${ }^{23}$ In Anbetracht des prekären Status des gesprochenen Standard ist diese Konzentration auf das Lautliche vielleicht etwas überraschend: Durrell, 'Standardsprache in England und Deutschland', zeigt, dass das für das Englische genau nicht so ist, es gibt also Standard mit Ausspracheunterschieden. Im Deutschen ist, wie mir scheint, diese Frage noch gar nicht so recht ausdiskutiert, weil die Natürlichkeit standardnahen Sprechens auch in den dialektfesten Gegenden erst ein relativ neues Phänomen darstellt.

${ }^{24}$ Variantenwörterbuch des Deutschen. Die Standardsprache in Österreich, der Schweiz und Deutschland sowie in Liechtenstein, Luxemburg, Osibelgien und Sülltrol, hg. v. Ulrich Ammon et al., Berlin / New York 2004. ${ }^{25}$ Am Beispiel des Buchstabens F wird das in Ludwig M. Eichinger, 'Deutsch in Österreich', German as a Foreign Language, 1/2005, 1 - 23: dargelegt <http://www.gfl-journal.de>. 
Noch deutlicher regional markiert ('Ruhrgebiet') sind ursprünglich andere Konstruktionen wie die $a m$-Verlaufsform, konkret ist heutzutage die Einschätzung dieser Formen im Einzelfall recht unterschiedlich, gewisse Verwendungen repräsentieren zumindest einen allgemein norddeutschen Substandard:

Er ist am Arbeiten (Er arbeitet gerade).

Dagegen sind Konstruktionen mit stärkerer syntaktischer Inkorporation deutlich regional und soziostilistisch markiert:

Wir waren das Haus am Bauen (Wir waren gerade dabei das Haus zu bauen/am Bauen des Hauses).

Beim Versuch entsprechend einen süddeutschen Substandard zu beschreiben, zeigt sich die Folge der Tatsache, dass im ober- und auch im mitteldeutschen Raum ein Kontinuum von der Hochsprache bis hin zu den örtlichen Dialekten besteht. Das hat im lautlichen Bereich zur Folge, dass die jeweiligen sprechsprachlichen Abweichungen direkter auf einzelregionale Formen Bezug nehmen als das für das hier angenommene 'Norddeutsche' der Fall ist. Das betrifft vor allem den Vokalismus und hat damit zu tun, dass das System der Vokalphoneme des Hochdeutschen (v. a. im Bereich der Langvokale und Diphthonge) einen systematischen Kompromiss aus den Gegebenheiten der hochdeutschen Dialekte darstellt! So kommt man auf dieser Ebene ohnehin immer schon zu kleinräumiger gekennzeichneten Regionalsprachen. Wenn so Besch analog den obigen Fällen von Berlinisch und Norddeutsch die 'zentralschwäbische Landschaftssprache des Raumes um Stuttgart' beschreibt, trifft das systematisch wie soziolinguistisch nicht dieselbe Ebene wie die oben an Berlin angelehnte Beschreibung: 'Beibehaltung der mittelhochdeutschen Diphthonge $i e, u o$, üe, also liob (lieb), guat (gut), miose (müssen) etc.; Vokalsenkung vor Nasalverbindung: fendo (finden) $[\ldots]^{26}$

Die weitgehende Abweichung dieser lautlichen Realisierungen von dem, was sich als Standard eingebürgert hat, ist auch den regionalen Sprechern weitgehend klar. Sie sind damit erste Wahl, wenn Merkmale auf dem Weg der Makrosynchronisation abgelegt werden. Dass diese Formen vom norddeutschen 'Durchschnittssprecher' bereits als 'Dialekt' bezeichnet werden, ist nicht überraschend: süddeutsche Substandardformen tragen notwendig in höherem Ausmaß Merkmale der regionalen Subgliederung in sich.

Dennoch gibt es auch Merkmale, die sich als Kennzeichen eines süddeutschen Standards betrachten lassen. Dazu gehört die stimmlose Aussprache des s-Lauts in anlautend prävokalischer und intervokalischer Position, die im wesentlichen das Mitteldeutsche mit umfasst, während die entsprechende Lenisierung anderer Fortes eher mit deutlicherer Südlichkeit (oberdeutsch) verbunden ist, insgesamt aber im gesamten Sprachgebiet sehr

${ }^{26}$ Besch, op.cit., S. 421 . 
stark vom Kontext des Plosivs (b, d, g) abhängig ist. Deutlich süddeutscher Substandard ist auch die [ig]-Aussprache von <-ig>. Merkmal eines süddeutschen Standards ist dann zudem nicht nur die Beibehaltung der Länge in den Standardfällen der Einsilbler (Tag, Rad) und das Fehlen der 'Auslautverhärtung' in diesen Fällen, sondern die Längung auch in der Norm gekürzter Wörter wie was und das. In einer Reihe nicht so systematisch einzusortierender Fälle geht der süddeutsche Substandard mit dem Österreichischen und Schweizerischen (etwa Kürze in der Endsilbe von Wörtern wie Kritik oder Fabrik). Hier herrscht aber insgesamt eine gewisse Unübersichtlichkeit:

Bei Erde, Jagd, Magd, Nische, hapern, Obst und vielleicht Städte teilt das Süddeutsche die norddeutsche Länge, dagegen geht es bei Afrika, Barsch, Harz, Nüstern, rösten und watscheln eher mit der österreichischen Kürze. ${ }^{27}$

Auch im grammatischen Bereich gibt es durchaus Süddeutschismen, der klassische Fall ist ja die Wahl des sein-Perfekts bei Verben wie stehen, gehen, sitzen, bei denen im süddeutschen Deutsch offenbar die Intransitivitätsregelung für die Wahl der Perfektform bestimmend ist, während der norddeutsche standardnahe Substandard hier eher nach semantischen Kriterien vorgeht.

Wir wollen es damit genug sein lassen. Erkennbar wurde, dass es auch im süddeutschen Raum standardnahe Substandardformen gibt, gegenüber denen aus den genannten Gründen die traditionell dialektalen und dialektnahen Formen in der gesellschaftlichen Kommunikation zurücktreten. Es ist offenkundig, dass diese Sprachformen auf dem Kontinuum zwischen den Dialekten und den standardnahen Substandards im süddeutschen Raum sozusagen beliebig mit Regionalitätsmarkern aufgefüllt werden können, da nicht mit einem echten Systembruch zu rechnen ist. Allerdings ist mit der Wahl von Optionen, in denen sich der schriftsprachliche Kompromiss des Neuhochdeutschen deutlich und erkennbar anders entschieden hat, eindeutig die Grenzen zu dialektnahen Substandards überschritten.

\section{2 ... und postmoderne Wahl}

Wir haben bisher davon gesprochen, wie sich Regionalität unter den gesellschaftlichen und nicht zuletzt medialen Bedingungen der zweiten Hälfte des zwanzigsten Jahrhunderts verändert hat, und dass das im Bereich der Öffentlichkeit gesprochene Deutsch - bei prinzipiell weiter vorhandenem regionalen Bezug - einerseits zu einer deutlich verstärkten Orientierung am Standard geführt hat. Das hatte andererseits zumindest in der allgemeinen Praxis dieser Sprachform zur Folge, dass der sprechsprachliche Charakter dieser Form erkennbarer wurde, was zum Einbezug von Elementen 
führte, die häufig in regional differenzierter Form vorlagen. Man muss der unbezweifelbaren Tatsache Rechnung tragen, dass wir seit einigen Jahrzehnten verstärkt in einer Gesellschaft leben, in der das Individuum weniger und weniger damit auskommt, die traditionellen Verhaltensweisen in wenn auch modifizierter Form fortzusetzen, sondern zu einer deutlich erhöhten Variation gedrängt ist, was eine deutliche Herausforderung, oft aber auch eine Chance darstellt. Wer nicht diese Variabilität zumindest in einem gewissen Ausmaß an den symbolischen Tag legen kann, dem sind bestimmte gesellschaftliche Beschränkungen auferlegt. Auf der Ebene der Standardsprache führt das dazu, dass sich eine nicht an traditionellen regionalen Untergliederungen orientierte Ebene von Kolloquialität entwickelt, die sich allerdings faktisch häufig als Ausbreitung vorhandener Formen beschreiben lässt. ${ }^{28}$

Zu der gesteigerten Variabilität gehört auch eine erhöhte sprachliche Variationsvielfalt, die auf jeden Fall eine aktive und passive Beherrschung der Hochsprache umfasst. Aber - und das ist eigentlich auch eine Konsequenz dieser Entwicklung - wer nur dieser Variante mächtig ist, hat auch ein Problem. Allerdings wäre der letzte Satz faktisch in den Konjunktiv zu setzen, da im Unterschied zum geschriebenen Standard, bei dem sich eher die Frage einer textsortenadäquaten Stilistik stellen mag, die Fragen der grammatischen Korrektheit aber weitestgehend geklärt sind. Selbst die zu erwartenden Varianten im Wortschatz und bei bestimmten Kollokationen stellen in dieser Hinsicht kein allzugroßes Problem dar. Insofern diese Varianz insgesamt eher allägliche Sachverhalte betrifft, die nun ihrerseits wieder in der Schriftlichkeit eine eher weniger bedeutsame Rolle spielen, ist sie für das durchschnittliche Schreiben eher ein minderes Problem. Interessant wird es allerdings auf der Ebene der gesprochenen Sprache. Hier hat sich in den letzten Jahrzehnten wohl die größte Verschiebung gegenüber den traditionellen Verhältnissen einer insgesamt regional geprägten Sprechsprachlichkeit ergeben. Die Sprachgebräuche in Sonderheit der jüngeren und vor allem jüngeren städtischen und gebildeten Sprecherinnen und Sprecher lassen häufig nur mehr an ganz marginalen Merkmalen - etwa relativ spezifischen Partikeln - die regionale Herkunft erkennen. Auf der anderen Seite wird damit aber die Frage systematisch drängender, wie denn standardgerechte Merkmale von Gesprochenheit aussehen, sofern der ideale Ort von Gesprochenheit nicht in den allerhöchst formellen Redeweisen sondern im Gespräch gebildeter Sprecher gesehen wird. Wenn man diese eher strukturelle Fragestellung für relevant und adäquat hält, hat man allerdings eigentlich auch eine Entscheidung über das normative Fundament unserer Sprechsprachlichkeit gefällt, das nicht unumstritten sein muss. Wenn man nicht die formellst denkbare Weise des Sprechens

${ }^{28}$ Das lässt sich etwa an clen Befunden des Atlas zur deutschen Alltagssprache (s. Stephan Elspaß, 'Variation and Change in Colloquial (Standard) German - The Atlas zur deutschen Alltagssprache (AdA) Project', in Fandrych und Salverda, op. cit., S. 201-16) sehen. 
sondern den gebildeten Dialog als Norm ansetzt, und wohlgemerkt nicht als empirische Schnittmenge des Vorfindlichen, sondern als Idealnorm, hat man gleichzeitig einer Hierarchisierung gesellschaftlicher Vorstellungen das Wort gesprochen, das mit der zentralen Stellung der historisch bildungsbürgerlichen Ideale und ihrer prinzipiellen Distanzsymbolik ein Ende macht. Wenn man diesen Überlegungen folgt, sind bestimmte Erscheinungen auf den verschiedenen linguistischen Ebenen, die vordem allenfalls als Phänomene einer gemäßigten Standardform gegolten hätten, zu zentralen Merkmalen einer gesprochenen Form und so zu etwas normativ Erstrebenswertem geworden. Es ist offenkundig, dass das unter den historischen Verhältnissen des Deutschen, die ich im ersten Teil dieses Beitrags skizziert habe, erst geschehen konnte, wenn solche Merkmale nicht als regionale und durch die regionalen Differenzen ggf. als soziale gelesen wurden, sondern sich als ein natürliches Merkmal von Kolloquialität verstehen lassen.

Dennoch spielt eine vorhandene oder auch nur anzitierte regionale Basis zumindest insofern eine Rolle, dass es schwerer als für den norddeutschen Raum zu entscheiden ist, wo ein süddeutsches Hochdeutsch, eine standardnahe Substandardvarietät, aufhört und die regionale Umgangssprache beginnt. Wenn man dieses Problem genereller angehen will, muss man sich fragen, welche Form ein regionaler Bezug in einer Gesellschaft unseres Typs sinnvollerweise annehmen könnte. Denn es ist ja nicht so, als gingen die regionalen Markierungen völlig verloren, sie setzen nur nicht traditionelle Unterteilungen fort. Vielmehr stellen regionale Markierungen in der Sprache eines der mehr oder minder frei zu wählenden Merkmale dar, die bei der symbolischen Konstitution der eigenen Identität genutzt werden. Da regionale Markierung lediglich eine der möglichen Symbolisierungskategorien ist, hat sie denn auch mit Hochsprachansprüchen, mit dem Image englischer Entlehnungen, allgemeinen Formen einer jugendlich geprägten Lockerheit und dergleichen zu kämpfen. ${ }^{29}$ Und so wird man denn zu beobachten haben, ob und wie sich Regionales in neuer Form in der Sprache niederschlägt. Wie das aussieht, kann dann je nach Umgebung und Lebensstil einigermaßen unterschiedlich sein. Das kann auch heißen ${ }^{30}$ dass sich neue Regionalitätsmerkmale in der Auswahl aus den Optionen moderner Standardsprachlichkeit entwickeln. Das ist sogar eher plausibel, wenn die normativen Vorstellungen über die Angemessenheit gesprochener Formen nicht mehr so sehr unmittelbar von der Schriftlichkeit kontrolliert-und wie man sagt, gebremst - werden, sondern die systemstabilisierenden Vorbilder in den sprechenden Medien, in Sonderheit im Fernsehen zu finden sind. Den Schluss, dass das so sei, muss man aus den empirischen Daten, die uns

${ }^{29}$ In diesen Kontext gehören gesellschaftliche Diskurse, wie sie Androutsopoulos, unter dem Titel 'Türk-Deutsch und pfälzischer Slang' beschreibt (vgl. Jannis Androutsopoulos, 'Ethnolekte in der Mediengesellschaft. Stilisierung und Sprachideologie in Performance, Fiktion und Metasprachdiskurs', in Fandrych und Salverda, op. cit. (S. 135ff.)).

${ }^{30}$ Ich verdanke diesen Hinweis einer mündlichen Bemerkung von Edgar Radtke, der solche Entwicklungen für das Italienische junger Sprecher nachweist. 
in diesem Feld zur Verfügung stehen, ziehen. ${ }^{31}$ Und er passt zu den verschiedenartigen soziologischen Analysen über die Verteilung sozialsymbolischer Symbolisierungsstrategien in der bundesdeutschen Gesellschaft - mit einer erheblichen Bedeutungszunahme einer freundlichen Mitte zuungunsten unfreundlicherer Ränder. Diese Aussage scheint einigermaßen unstrittig, was immer man ansonsten von der Gesamterklärungsreichweite dieser kultursoziologischen Konzepte halten mag. Es gibt eine Reihe von neueren Untersuchungen, von denen die Annahme eines solchen Trends gestützt wird. Sie zeigen im wesentlichen, dass modernes standardsprachliches Sprechen davon lebt, dass sich bestimmte Ausprägungen sprechsprachlicher Optionen großräumiger über das Land verteilen. Es ist zu kurz gegriffen, wenn man darin die Dominantsetzung des einen oder anderen alten Sprachraums sieht, vielmehr geht es um die Entwicklung einer Sprechebene, die mit dem jeweils persönlich intendierten sozialen Bild verträglich ist, und sich dazu möglichst unauffälliger Mittel bedient. In der sprachlichen und gesellschaftlichen Gegenwart führt das dazu, dass die gesprochenen Formen regionaler Begrenzung in Richtung eines gesprochenen Standards ausgeglichen werden, bzw. in der Verwendung als markiert zu beurteilen sind. Das gilt ganz sicher für die als normverträglich gehaltene Aussprache in kritischen Bereichen. Da gerade die neuen sprechsprachlichen Dinge erst allmählich ihren Systemstatus stabilisieren, spielen hier Übereinkünfte über normative Erwartungen eine erhebliche Rolle, und es ist eine noch nicht endgültig zu beantwortende Frage, welchen Geltungsbereich ein entsprechender Konsens jeweils hat. ${ }^{32}$

Nun kann man auf der einen Seite ganz deutlich sehen, dass dabei bestimmte Elemente - etwa eine Reihe von Partikeln - ihre traditionelle regionale Färbung verlieren. Man kann das etwa schön an der Verteilung der Partikeln halt und eben sehen, die traditionell als südlicher oder nördlicher Typ nebeneinander standen, jetzt aber im ganzen Sprachraum verbreitet sind, was deswegen ganz gut geht, weil sich ihr Nebeneinander in eine funktionale Differenz umsetzen lässt. Das in seiner impliziten Festlegung grundsätzlichere eben, das gut zum Modell einer nördlichen Distanzhöflichkeit passt, steht nun neben dem 'weicheren' halt, das zu einem Nähesystem von Höflichkeit stimmt, wie es traditionell eher mit südlichen Traditionen des Sprechens verbunden wird. Das Ausgreifen dieser Form kann man als Indiz lesen, dass kommunikativ nachgiebigere Strategien des Sprechens (in

${ }^{31}$ Ich meine dabei in Sonderheit die ersten Interpretationen der im Rahmen des Projekts 'Deutsch heute' am IDS erhobenen Materiatien gesprochenen Standards in zwei Altersgruppen; vgl. Ralf Knöbl, Stefan Kleiner, Caren Brinkmann und Nina Berend, 'German Today - An extensive speech data collection in the German speaking area of Europe', in Procedings of the 4th Corpus Linguistics Conference, Birmingham $2007<\mathrm{http}$ ///corpus, bham,ac.uk/corplingproceedings07/paper/ 136_Paper.pdf>.

${ }^{32}$ Was ist das Kollektiv der 'kollektiven Vorstellungen', die Langer zitiert (vgl. Nils Langer, 'Finding Standard German - Notes on Linguistic Codification', in Fandrych und Salverda, op. cit., S. 217 - 40 (S. 237))? 
schriftsprachnaher Form) in der heutigen bundesdeutschen Gesellschaft insgesamt an Raum gewonnen haben - was unmittelbar zu kultursoziologischen Einschätzungen der sozialsymbolischen Bedeutung verschiedener Milieus passen würde. ${ }^{33}$

${ }^{33}$ Das spiegelt sich auch in der relativen Stärke der 'bürgerlichen Mitte' wider, wie sie sich aus den Sinus-Studien der letzten Jahre ergibt (vgl. <http://www.sinus-sociovision.de>) . 